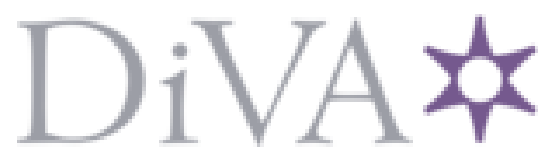

http://www.diva-portal.org

This is the published version of a paper published in Journal of Chemical Physics.

Citation for the original published paper (version of record):

Eland, J., Hochlaf, M., Linusson, P., Andersson, E., Hedin, L. et al. (2010)

Triple ionization spectra by coincidence measurements of double Auger decay: The case of OCS.

Journal of Chemical Physics, 132(1): 014311

http://dx.doi.org/10.1063/1.3274648

Access to the published version may require subscription.

N.B. When citing this work, cite the original published paper.

Permanent link to this version:

http://urn.kb.se/resolve?urn=urn:nbn:se:uu:diva- 121200 


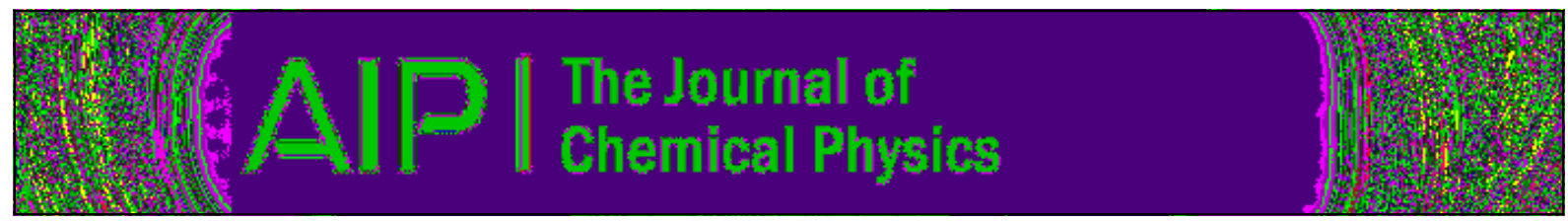

Triple ionization spectra by coincidence measurements of double Auger decay: The case of OCS

J. H. D. Eland, M. Hochlaf, P. Linusson, E. Andersson, L. Hedin, and R. Feifel

Citation: The Journal of Chemical Physics 132, 014311 (2010); doi: 10.1063/1.3274648

View online: http://dx.doi.org/10.1063/1.3274648

View Table of Contents: http://scitation.aip.org/content/aip/journal/jcp/132/1?ver=pdfcov

Published by the AIP Publishing

\section{Articles you may be interested in}

Dissociative double-photoionization of butadiene in the 25-45 eV energy range using 3-D multi-coincidence ion momentum imaging spectrometry

J. Chem. Phys. 143, 114309 (2015); 10.1063/1.4931104

Three body dissociation of CS $22+$ subsequent to various S(2p) Auger transitions

J. Chem. Phys. 139, 164309 (2013); 10.1063/1.4826468

Spectra of the triply charged ion CS $23+$ and selectivity in molecular Auger effects

J. Chem. Phys. 132, 104311 (2010); 10.1063/1.3352549

Multielectron coincidence spectroscopy for core-valence doubly ionized states of CO

J. Chem. Phys. 127, 044305 (2007); 10.1063/1.2750341

Ab initio simulation of molecular Auger spectra: Nuclear dynamics effects in the spectra of carbonyl sulfide J. Chem. Phys. 107, 6070 (1997); 10.1063/1.474275

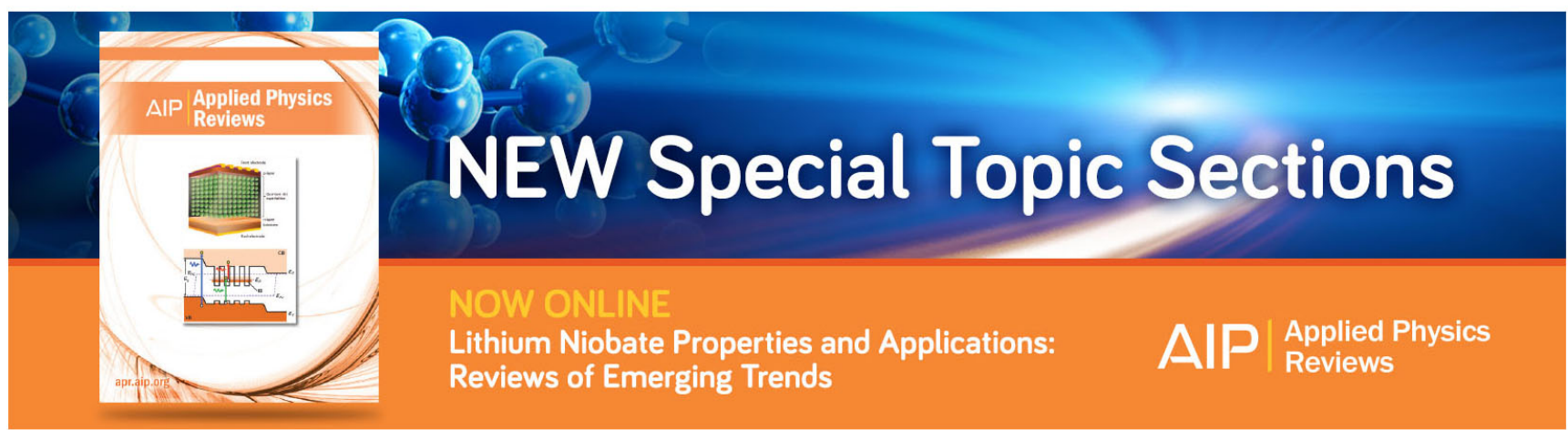




\title{
Triple ionization spectra by coincidence measurements of double Auger decay: The case of OCS
}

\author{
J. H. D. Eland, ${ }^{1}$ M. Hochlaf, ${ }^{2, a)}$ P. Linusson, ${ }^{3}$ E. Andersson, ${ }^{4}$ L. Hedin, ${ }^{4}$ and R. Feifel ${ }^{4, b)}$ \\ ${ }^{1}$ Department of Chemistry, Physical and Theoretical Chemistry Laboratory, Oxford University, \\ South Parks Road, Oxford OX1 3QZ, United Kingdom \\ ${ }^{2}$ Laboratoire Modélisation et Simulation Multi Echelle, MSME UMR 8208 CNRS, Université Paris-Est, \\ 5 boulevard Descartes, 77454 Marne-la-Vallée, France \\ ${ }^{3}$ Department of Physics, Stockholm University, AlbaNova University Centre, SE-106 91 Stockholm, Sweden \\ ${ }^{4}$ Department of Physics and Materials Science, Uppsala University, P.O. Box 530, SE-751 21 \\ Uppsala, Sweden
}

(Received 16 September 2009; accepted 24 November 2009; published online 7 January 2010)

\begin{abstract}
By combining multiple electron coincidence detection with ionization by synchrotron radiation, we have obtained resolved spectra of the $\mathrm{OCS}^{3+}$ ion created through the double Auger effect. The form of the spectra depends critically on the identity of the atom bearing the initial hole. High and intermediate level electron structure calculations lead to an assignment of the resolved spectrum from ionization via the S $2 p$ hole. From the analysis it appears that the double Auger effect from closed shell molecules favors formation of doublet states over quartet states. Molecular field effects in the double Auger effect are similar to those in the single Auger effect in linear molecules. (C) 2010 American Institute of Physics. [doi:10.1063/1.3274648]
\end{abstract}

\section{INTRODUCTION}

The triply charged ion $\mathrm{OCS}^{3+}$ is known to be stable on the mass spectrometer time scale of a few microseconds. ${ }^{1}$ Its spectrum is entirely unknown but is expected to be complex with a high density of electronic states. In this paper we report triple ionization spectra of the molecule obtained by coincidence methods combined with the use of synchrotron radiation to initiate double Auger decays from inner shell holes located on the different atoms.

In the double Auger process, decay of an excited singly charged ion with an inner shell hole causes emission of two further electrons. One outer electron falls into the hole, releasing more than sufficient energy for ejection of one, two, or even more outer electrons. In the single Auger process, where only one new electron is ejected, the Auger electron has a definite energy corresponding to the difference in energy between the initial hole and the final doubly charged ion state. Spectra of molecular doubly charged ions have been known for many years from study of Auger electron spectra. In double Auger, by contrast, the two Auger electrons share the available excess energy, dividing it between them with a distribution, which is in principle unknown. Recent coincidence studies of the double Auger effect in atoms ${ }^{2-5}$ have shown that both "direct" and "cascade" pathways exist, giving continuous and discrete electron distributions, respectively. Cascade pathways involve the formation of intermediate doubly ionized states with quantized energies and measurable widths (lifetimes). In double Auger, as in single Auger spectroscopy, the broadening effect of the width of the hole states can be eliminated by coincidence measurement of

\footnotetext{
${ }^{a)}$ Electronic mail: majdi.hochlaf@univ-mlv.fr.

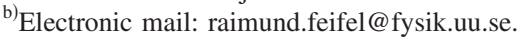

the energies of all the emitted electrons, both primary (photoelectrons) and secondary (Auger electrons). ${ }^{6,7}$

To characterize and where possible to identify the states of the molecular triply charged ions $\mathrm{OCS}^{3+}$ revealed in the spectra, we have carried out both simple and high level quantum chemical calculations. As for all doubly charged molecular ions ${ }^{8}$ including the related case of $\mathrm{OCS}^{2+}$ dication, ${ }^{9}$ accurate energy calculations require full accounting for electron correlation, as many of the states have strongly mixed electron configurations.

\section{EXPERIMENTAL}

\section{A. Experimental methods}

To obtain spectra of the triply charged ions, we measure, in coincidence, the energies of all three electrons and in some experiments also the parent ions created in photoionization by the double Auger effect. This is done using a magnetic bottle time-of-flight (TOF) analyzer installed on the soft-x-ray undulator beamline U49/2 PGM2 at the electron storage ring BESSY-II in Berlin. The accessible photon energies at this beamline range from 85 to over $1000 \mathrm{eV}$, encompassing the inner shell energies of $\mathrm{C}(1 \mathrm{~s}), \mathrm{O}(1 \mathrm{~s})$, and $\mathrm{S}$ (2s and 2p).

The magnetic bottle spectrometer has been described before. ${ }^{10}$ Briefly, ionization occurs where the light beam crosses the effusive target gas jet from a hollow needle of 1 $\mathrm{mm}$ internal diameter. This source is located within the strongly divergent magnetic field (approximately $0.7 \mathrm{~T}$ ) from a conical permanent magnet, which reflects and directs almost all emitted electrons into a $2.2 \mathrm{~m}$ long flight tube, where they follow the field lines of a weak $\left(10^{-3} \mathrm{~T}\right)$ solenoid. At the end of the flight tube they impinge on a microchannel plate detector containing three plates in Z-stack con- 


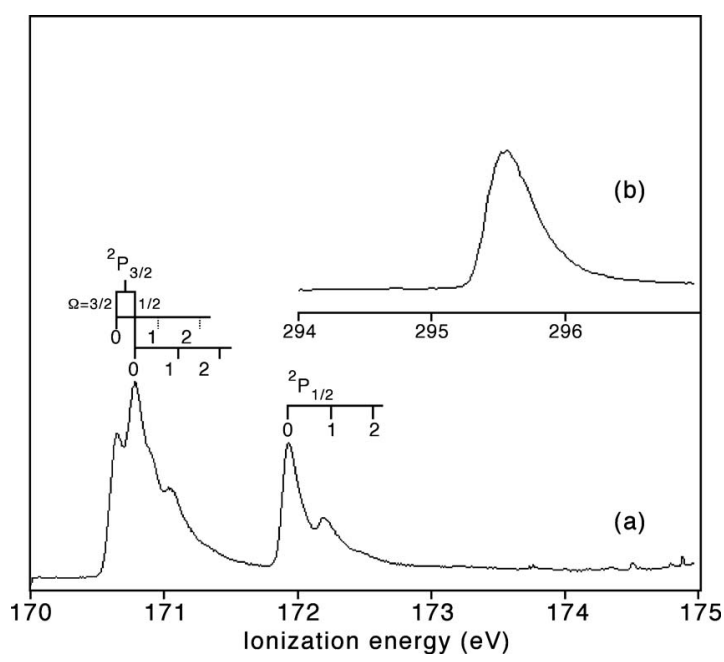

FIG. 1. Photoelectron spectra (a) of the S 2 p hole states at $175 \mathrm{eV}$ photon energy and (b) of the $\mathrm{C}$ 1s hole at $305 \mathrm{eV}$. Peak distortion due to PCI is apparent. The small peaks at the bottom right in (a) are spurious except for the slightly broader one at $174.5 \mathrm{eV}$ as explained in the text.

figuration. The electron signals are recorded, after amplification and discrimination, at a multichannel multihit time-to-digital converter in a local computer. The converter also registers timing signals from the storage ring. In this mode of operation the energy resolution for single electrons can be approximated as one part in 50 of the electron energy.

In the configuration used to detect ions as well as electrons, ${ }^{4,11}$ the conical magnet is replaced by an assembly of ring magnets and grids, which allows ions to be extracted in the opposite direction to electrons, into a simple TOF mass spectrometer, which is also equipped with a microchannel plate detector. The grids can be polarized either to extract ions with good mass resolution using the electrons only to provide timing information or to detect both ions and electrons simultaneously but with less good resolution for both particles. For electrons the resolution under these conditions is approximately one part in 20 of the electron energy.

\section{B. Experimental data analysis}

Because the period between ionizing light pulses from the storage ring of $800.5 \mathrm{~ns}$ is often shorter than the flight time of slow electrons in our apparatus, typically $5000 \mathrm{~ns}$ for zero initial energy, the flight times of detected electrons are ambiguous. The true flight times may exceed the measured ones by an integer number of periods, including zero. This ambiguity can be completely resolved in the Auger effects by choosing a photon energy so high that the photoelectron always arrives either before any Auger electron or within a single pulse period. Unfortunately this strategy leads to poor resolution for the photoelectron itself and so entails loss of selectivity and, since all three electron energies are added together, poor resolution in the final spectrum. In this work we have generally used photon energies within $10 \mathrm{eV}$ of the inner shell edges, where the photoelectron resolution is good (e.g., Fig. 1, above). Under these conditions the desired Auger processes are identified and calibrated by selecting events with one electron at an energy in the peak of the photoelectron signal, as correctly timed for its known energy. This choice alone selects all Auger events where one Auger electron flight time is within a single period, that is, where the fastest Auger electron's energy is more than $21 \mathrm{eV}$. In addition, and before this selection, if any electron of a triple has an apparent energy too high to be possible by energy conservation even as a direct ionization, one ring period is added to the timing of all three electrons. The combined strategy then correctly calibrates the vast majority of double Auger events and is absolutely sufficient for the spectra of interest at relatively low triple ionization energy. This is because any residual incorrectly timed events have total Auger electron energies of less than $10 \mathrm{eV}$ and so can contribute only to remote parts of the spectra at binding energies just below the initiating edge.

In addition to true triple ionizations, the data contain a small number of false coincident electron triples whose members do not all originate in the same ionizing light pulse. The majority of these interlopers comes from double ionizations plus one extra electron from a later light pulse. Their number is kept very small by using a low (approximately $3000 \mathrm{~s}^{-1}$ ) total electron count rate, and they have no significant effect on the spectra. Their relative contribution can be judged approximately from the areas of sharp low energy peaks in the photoelectron spectrum (Fig. 1).

\section{THEORETICAL METHODS}

We performed our electronic calculations using the MOLPRO program suite ${ }^{12}$ in the $\mathrm{C}_{2 \nu}$ and $\mathrm{C}_{\mathrm{s}}$ point groups. The $\mathrm{O}$, $\mathrm{C}$, and $\mathrm{S}$ atoms are described using either the aug cc-pVQZ or the aug cc-pV5Z basis sets. ${ }^{13,14}$ For electronic excited states pattern and evolutions, we used the complete active space self-consistent field (CASSCF) (Ref. 15) method, followed by the internally contracted multireference configuration interaction (MRCI) ${ }^{16,17}$ approach. In these calculations, electronic states having the same spin multiplicity are averaged together using the MOLPRO averaging procedure. All valence molecular orbitals are optimized and all electrons are correlated. For MRCI, all the configurations having a weight greater than 0.01 in the CI expansion of the CASSCF wave functions are taken into account as a reference. This weight is the size of the reference space CI coefficient. The calculations were done in $\mathrm{C}_{2 \nu}$ symmetry with wave functions corresponding to more than $4.9 \times 10^{8}$ and $7.4 \times 10^{8}$ uncontracted configurations for the doublets and the quartets, respectively. For the geometry optimizations, we used CASSCF method and RCCSDT method (see e.g., Ref. 18); the latter implies a restricted coupled-cluster with single and double and perturbative triple excitations.

\section{EXPERIMENTAL RESULTS}

\section{A. Ion mass and coincidence spectra of OCS at different photon energies}

At all photon energies below the onset of inner shell excitation, the mass spectrum of OCS is dominated by single ionization. At $160 \mathrm{eV}$, for example, single ionization makes up about $85 \%$ of the total with $15 \%$ double ionization and a negligible fraction of triple ionization. At $180 \mathrm{eV}$ photon energy, just above the $\mathrm{S} 2 \mathrm{p}$ edges, by contrast, double ion- 


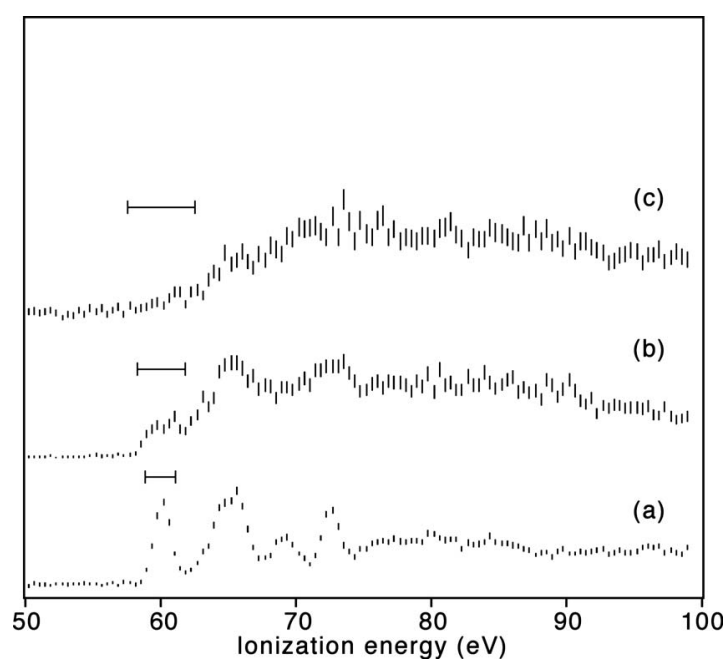

FIG. 2. Triple ionization spectra of OCS by double Auger (a) via the S $2 p$ edge with $175 \mathrm{eV}$ photons, (b) via the $\mathrm{S} 2$ s edge with $305 \mathrm{eV}$ photons, and (c) via the $\mathrm{C} 1 \mathrm{~s}$ edge also with $305 \mathrm{eV}$ photons.

ization makes up $43 \%$ and triple ionization about $2 \%$. The triply charged parent ion is clearly visible in the mass spectrum but represents only a few percent of overall triple ionization. Major dissociative triple ionizations produce $\mathrm{O}^{+}$ $+\mathrm{CS}^{2+}$ and $\mathrm{S}^{2+}+\mathrm{CO}^{+}$, but a full analysis of all the dissociative triple ionization pathways is not possible because of the indistinguishability of $\mathrm{S}^{2+}$ and $\mathrm{O}^{+}$ions at our mass spectral resolution. At even higher photon energies, above the $\mathrm{C} 1 \mathrm{~s}$ and $\mathrm{O} 1 \mathrm{~s}$ edges, triple ionizations including the $\mathrm{OCS}^{3+}$ ion are even more abundant relative to the lower degrees of ionization $\left(>6 \% \mathrm{OCS}^{3+}\right.$ at 310 and $\left.550 \mathrm{eV}\right)$. It seems clear that the Auger effects produce multiple photoionization more effectively in this molecule than do the photoionization processes not involving inner shells.

\section{B. Photoelectron spectra}

Photoelectron spectra of the S 2p and C 1s hole states measured at 175 and $305 \mathrm{eV}$ as spectra of the second electrons in pairs are shown in Fig. 1. Like the $\mathrm{C} 1 \mathrm{~s}$ band, the $\mathrm{S}$ $2 \mathrm{~s}$ band (not shown) has no detectable fine structure and is of very low intensity at all photon energies. The $S 2 p$ ionization spectrum is split into $\mathrm{J}=3 / 2$ and $\mathrm{J}=1 / 2$ by the spin-orbit interaction $(1.29 \mathrm{eV})$; then the ${ }^{2} \mathrm{P}_{3 / 2}$ term is split further by $0.14 \mathrm{eV}$ into $\Omega=3 / 2(\Pi)$ and $\Omega=1 / 2(\Sigma)$ components by the molecular (ligand) field. Each of the three resulting states shows vibrational structure. The vibration interval of $0.26 \mathrm{eV}$ (2100 $\mathrm{cm}^{-1}$ ) clearly identifies this as the mode $\nu_{1}$, which is mainly a $\mathrm{C}-\mathrm{O}$ stretch. Excitation of the lower frequency modes ( 0.11 and $0.064 \mathrm{eV}$ in the neutral molecule) may be concealed by the lifetime broadening and postcollision interaction (PCI) distortion of the lines. The widths (full width at half maximum) of the best resolved lines are about $0.13 \mathrm{eV}$, which would correspond to a natural lifetime of $85 \mathrm{fs}$. The line positions measured here $[\mathrm{S} 2 \mathrm{p}, \Omega=3 / 2(\mathrm{v}=0)$ at 170.6 $\mathrm{eV}, \Omega=1 / 2(\mathrm{v}=0)$ at $171.9 \mathrm{eV}, \mathrm{S} 2 \mathrm{~s}$ at $235 \mathrm{eV}$, and $\mathrm{C} 1 \mathrm{~s}$ at $295.5 \mathrm{eV}]$ are in agreement with previous measurements. ${ }^{19}$

In addition to the main peaks, some small sharp features can be seen at high ionization energy (low electron energy)

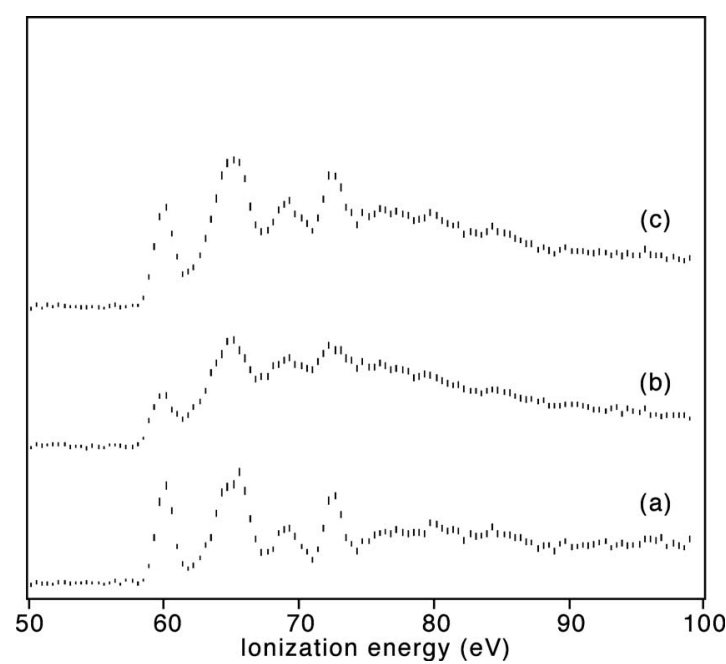

FIG. 3. Triple ionization spectra of OCS by double Auger via S 2p (a) taking Auger electrons of $>10 \mathrm{eV}$ only (b) taking Auger pairs only with one below $10 \mathrm{eV}$, and (c) taking all Auger pairs with no discrimination.

in the spectrum of S 2p. Most of these are spurious, arising from later light pulses. Only the slightly broader peak at an apparent binding energy of $174.5 \mathrm{eV}$ is real. It comes from autoionization of superexcited atomic oxygen created in dissociative ionization processes and is discussed further below.

\section{Spectroscopy of the $\mathrm{OCS}^{3+}$ ion}

We have obtained spectra of this triply charged ion by detecting, in coincidence, all three electrons from the photon-induced double Auger effect via holes in the $S 2 p, S$ $2 \mathrm{~s}$, and $\mathrm{C} 1 \mathrm{~s}$ shells at various photon energies. The energy of the $\mathrm{O}$ 1s edge is so high that no useful resolution could be obtained by our present experimental method. As in the related $\operatorname{cases}^{20}$ of $\mathrm{CO}_{2}$ and $\mathrm{CS}_{2}$, the form of the spectra depends strongly on the identity of the initial hole state and on selection within the distribution of excess energy between the two outgoing Auger electrons.

Figure 2 shows spectra of $\mathrm{OCS}^{3+}$ originating from the production of holes at the $\mathrm{S} 2 \mathrm{p}, \mathrm{S} 2 \mathrm{~s}$, and $\mathrm{C} 1 \mathrm{~s}$ edges, all three involving the constraint that only Auger electrons with more than $10 \mathrm{eV}$ energy are accepted. The reason for this choice will be explained below. The estimated instrumental energy resolution of each spectrum is also indicated, as this greatly affects their appearance. The spectra from $S 2 p$ and $S$ $2 \mathrm{~s}$ are quite similar in showing the same (partially) resolved bands, though with somewhat different relative intensities. The C 1s spectrum is different, with its peak intensity displaced toward higher ionization energy and almost no intensity in the first band. From these spectra we infer that the initial hole location is an important factor determining the form of the spectra, as Auger electrons are ejected preferentially from orbitals located on the atom bearing the initial hole. The orbital character (s type, p type, etc.) of the hole may also have an effect, but this is less clear as the total energy available to the two Auger electrons may also play a part. The lowest triple ionization energy of OCS is found to be $60 \pm 0.25 \mathrm{eV}$.

As in atomic and molecular double photoionization, ${ }^{21,22}$ 


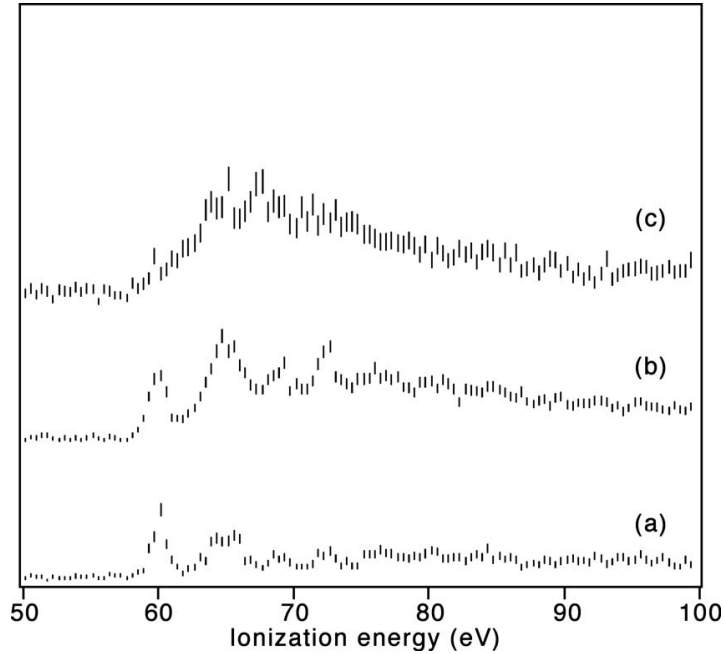

FIG. 4. Triple ionization spectra of OCS by double Auger from selected parts of the $S 2$ p photoelectron spectrum [Fig. 1(a)]. (a) from the $\Omega=3 / 2$ (II) $(\nu=0)$ component of the ${ }^{2} \mathrm{P}_{3 / 2}$ initial hole; (b) from the $\Omega=1 / 2(\Sigma)$ $(\nu=0)$ peak; (c) from all the main hole states but also including one electron of $0.3-0.5 \mathrm{eV}$ from autoionization of atomic oxygen.

the two Auger electrons emitted in double Auger share the available energy in a distribution peaking at the extremes (a peak near zero, the complement near maximum energy) and a broad valley between. No sharp intermediate peaks, which would be characteristic of cascade Auger processes, are seen in the distributions for formation of the lowest state of $\mathrm{OCS}^{3+}$ by double Auger, but broad peaks could not be detected easily and may contribute at low electron energy. The best resolved triple ionization spectra are obtained by requiring that both Auger electrons have a minimum energy of 5 $\mathrm{eV}$ or more, so excluding the extreme asymmetric energy sharing. Spectra illustrating this are shown together in Fig. 3. For the bottom spectrum (a), events where the lowest Auger electron energy was above $10 \mathrm{eV}$ were selected. For spectrum (b) the slowest Auger electrons had less than $10 \mathrm{eV}$, while the uppermost spectrum (c) involves no selection and is equal to the sum of (a) and (b). The significantly better sharpness of spectrum (a) is apparent, but the reason for it is not. One definite contribution is the electron energy resolution, which is at its worst in asymmetric sharing as one electron has the maximum possible energy. In this case the instrumental width must be at least $(170-60) / 50=2.2 \mathrm{eV}$ but may be worse because for very high energy electrons the width goes as $\mathrm{E}^{3 / 2}$, not directly as $\mathrm{E}$. The width of the first band in spectrum (b) is about $2.5 \mathrm{eV}$. In more symmetric sharing, if the valley part of the Auger electron distribution is flat, the average energy of the faster electron would give an estimated instrumental width of the order of $1.1 \mathrm{eV}$, rather close to the observed width of the first peak in spectrum (a) of $1.3 \mathrm{eV}$. Thus the differing sharpness of bands in spectra (a) and (b) can probably be accounted for entirely on the basis of instrumental resolution. However, even visual inspection shows that convolution of spectrum (a) with a broadening function will never match spectrum (b) in intensity distribution. There is therefore an additional principle at work, which may be a contribution of cascade processes to the low energy electron peak.
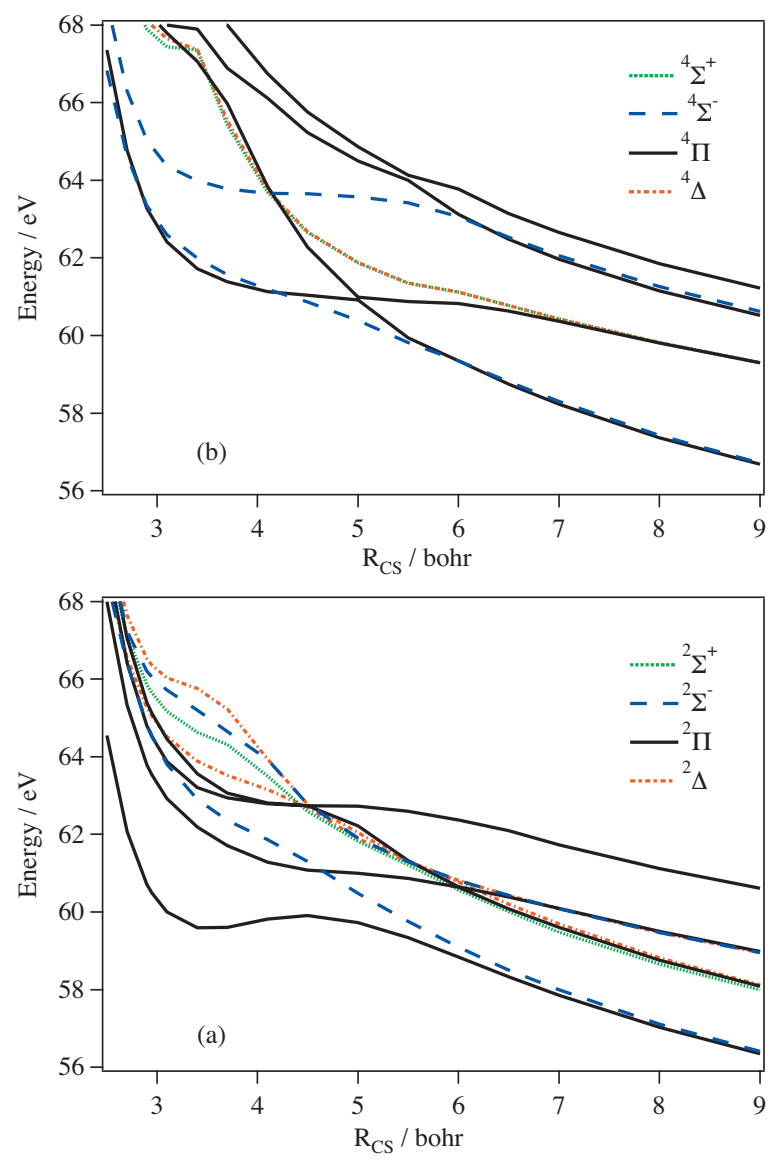

FIG. 5. MRCI/CASSCF collinear potential energy curves of the doublet and quartet electronic states of $\mathrm{OCS}^{3+}$ along the $\mathrm{R}_{\mathrm{CS}}$ coordinate. The $\mathrm{R}_{\mathrm{CO}}$ distance is set to 2.175 bohr. These curves are given in energy with respect to the OCS $\mathrm{X}^{1} \Sigma^{+}$minimum energy.

In the spectra exhibited so far, all the hole states produced by $\mathrm{S} 2 \mathrm{p}$ photoelectron ejection have been included in deriving the double Auger spectra. However, within the multidimensional coincidence data, it is easy to select spectra arising from any individual part of the photoelectron distributions in Fig. 1, excluding the rest. For instance, we can choose the ${ }^{2} \mathrm{P}_{3 / 2}$ and ${ }^{2} \mathrm{P}_{1 / 2}$ levels individually, individual ligand field components, individual vibrations, or different parts of the PCI-broadened distributions. All of these selections produce some contrasts in the triple ionization spectra, but most are very minor. By far the most significant contrast is between the molecular ligand field-split components $\Omega$ $=3 / 2(\Pi)$ and $\Omega=1 / 2(\Sigma)$ of the ${ }^{2} \mathrm{P}_{3 / 2}$ complex, as illustrated in Fig. 4. The difference in relative band intensities [spectra (a) and (b) of Fig. 4] is not entirely unexpected, as discussed in a later section. Figure 4 also contains a third spectrum, (c), which has been derived by taking both a photoelectron and one electron in the energy range $0.3-0.5 \mathrm{eV}$, corresponding to one of the small peaks in Fig. 1(a). Spectrum (c) shows states of $\mathrm{OCS}^{3+}$, which dissociate initially to doubly charged products including a metastable $\mathrm{O}^{*}$ atom, which later autoionizes producing a $0.4 \mathrm{eV}$ electron. The same atomic oxygen autoionization has been observed extensively before in single and double ionizations of small oxygen-containing molecules. ${ }^{22,23}$ This dissociation evidently happens predominantly from higher states of the triply charged ion and hardly, if at all, from the lowest state. 

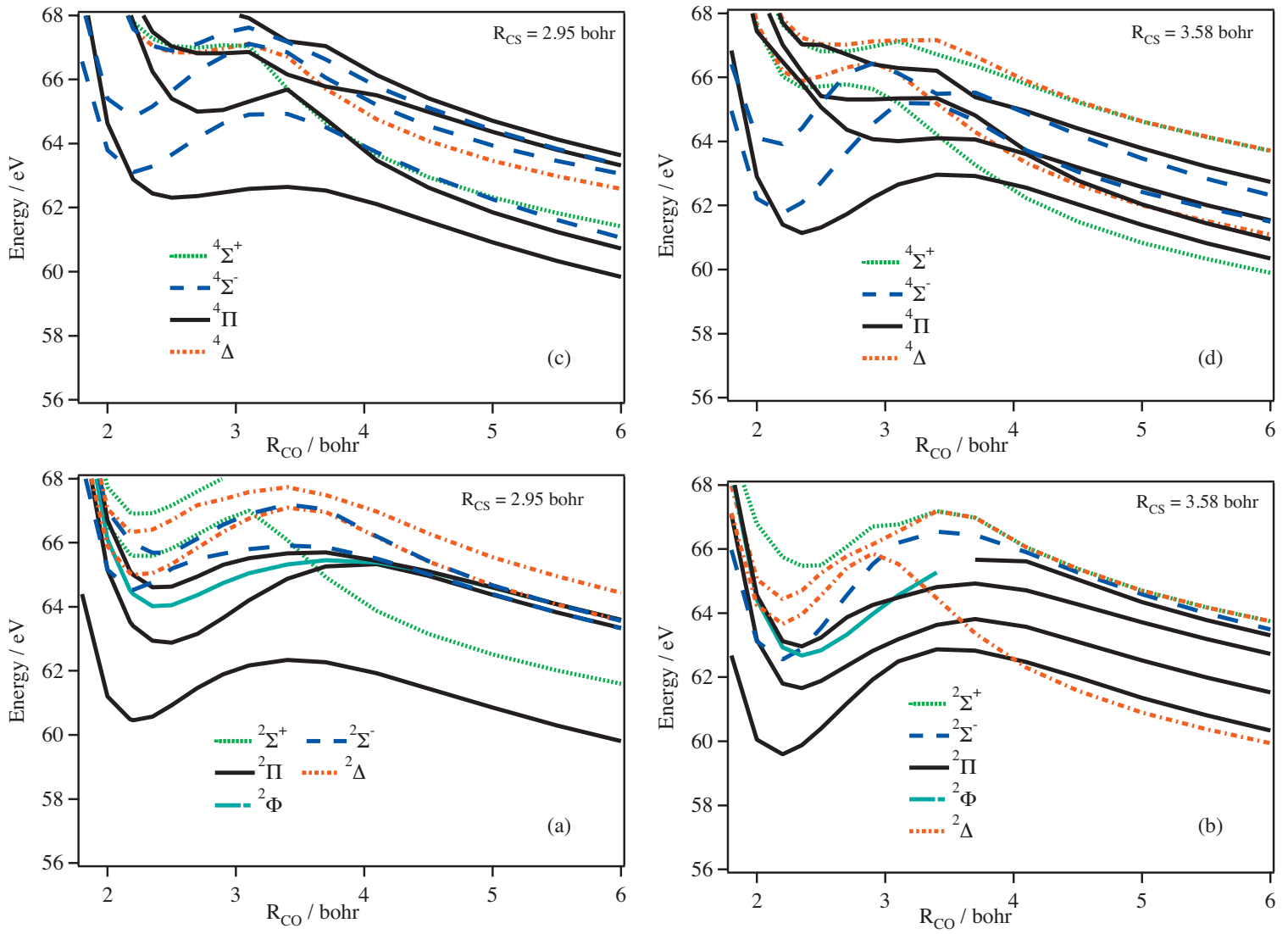

FIG. 6. MRCI/CASSCF collinear potential energy curves of the doublet and quartet electronic states of OCS ${ }^{3+}$ along the $\mathrm{R}_{\mathrm{CO}}$ coordinate. In the left panel the $\mathrm{R}_{\mathrm{CS}}$ distance is set to 2.95 bohr. In the right panel the $\mathrm{R}_{\mathrm{CS}}$ coordinate is kept fixed to 3.58 bohr. See text for more details. These curves are given in energy with respect to the OCS $\mathrm{X}^{1} \Sigma^{+}$minimum energy.

To examine which states dissociate and which do not, we could also gather fourfold coincidences between three electrons and an $\mathrm{OCS}^{3+}$ ion under the low resolution conditions mentioned in the Experimental section. Because of the low collection efficiency for ions (approximately 10\%; cf. 60\% for electrons) and also the limited available runtime, the number of counts accumulated in this mode was very small. As a result the spectrum obtained has very poor statistics; the best that can be said is that it is consistent with the idea that only the ground state of $\mathrm{OCS}^{3+}$ is stable, as is indicated by the theoretical calculations.

\section{THEORETICAL RESULTS}

The electronic orbital structure of OCS can be written as $\mathrm{S} 1 \mathrm{~s}^{2} \mathrm{O} 1 \mathrm{~s}^{2} \mathrm{C} 1 \mathrm{~s}^{2} \mathrm{~S} 2 \mathrm{~s}^{2} \mathrm{~S} 2 \mathrm{p}^{6} 6 \sigma^{2} 7 \sigma^{2} 8 \sigma^{2} 9 \sigma^{2} 2 \pi^{4} 3 \pi^{4}$.

Vertical ionization energies from the outermost orbitals are well known from the photoelectron spectrum of the molecule ${ }^{24}$ and can be crudely interpreted as orbital energies. From this orbital configuration as confirmed by earlier calculations, it is expected that the ground state of $\mathrm{OCS}^{3+}$ is the ${ }^{2} \Pi$ state from removal of three electrons from the outermost $(3 \pi)$ orbital. This is confirmed by full calculations at the RCCSD(T)/aug cc-pVXZ, CASSCF/aug cc-pVXZ (X $=Q, 5)$ levels of theory. These calculations show that all vibrational wavenumbers are positive.

At the highest (RCCSD(T)/aug cc-pV5Z) level of theory, the triple ionization energy is calculated to be $59.59 \mathrm{eV}$, in excellent agreement with observation. The optimized structure of the ion has bond lengths $\mathrm{R}_{\mathrm{C}-\mathrm{O}}=1.14 \AA$ and $\mathrm{R}_{\mathrm{C}-\mathrm{S}}$ $=1.84 \AA$, which differ somewhat from the experimental neutral ground state distances of 1.160 and $1.560 \AA$. The $\mathrm{C}-\mathrm{O}$ bond length is almost unchanged, while the $\mathrm{C}-\mathrm{S}$ bond lengthens, which is consistent with removal of all three electrons from the outermost $\pi$ orbital, located almost entirely on the $\mathrm{S}$ atom.

We have also calculated the energies of all states up to $15 \mathrm{eV}$ above the threshold at the CASSCF and MRCI levels using the bond lengths characteristic of the neutral molecules. For the lower-lying state up to $4.5 \mathrm{eV}$ above threshold, we have calculated the state energies and potential energy curves at the CASSCF/aug cc-pVQZ level. These data are given in Table I.

The barrier to dissociation by lengthening the $\mathrm{C}-\mathrm{S}$ bond on the ground state surface is about $0.3 \mathrm{eV}$, which suggests that only the lowest vibration level of the ion is likely to survive even for the few microseconds needed for mass spectrometric detection. Potential energy curves for all the states up to about $5 \mathrm{eV}$ above the ground state have been calculated and are shown in Figs. 5-7. Since the equilibrium geometry of the inner shell hole molecular state is not trivial to predict, we computed two sets of potentials where the $\mathrm{R}_{\mathrm{CO}}$ distance is set to 2.175 bohr $\left(\sim \mathrm{R}_{\mathrm{e}, \mathrm{CO}}\right.$ OCS X) and the $\mathrm{R}_{\mathrm{CS}}$ coordinate is kept fixed to either $2.98 \mathrm{bohr}\left(\sim \mathrm{R}_{\mathrm{e}, \mathrm{CO}}\right.$ OCS X) or $3.58 \mathrm{bohr}$ 
TABLE I. Dominant electron configuration and vertical excitation energies of the doublet and quartet electronic states of OCS ${ }^{+++}$lying in the $0-15 \mathrm{eV}$ internal energy domain. These data are quoted at equilibrium geometry of the neutral OCS X.

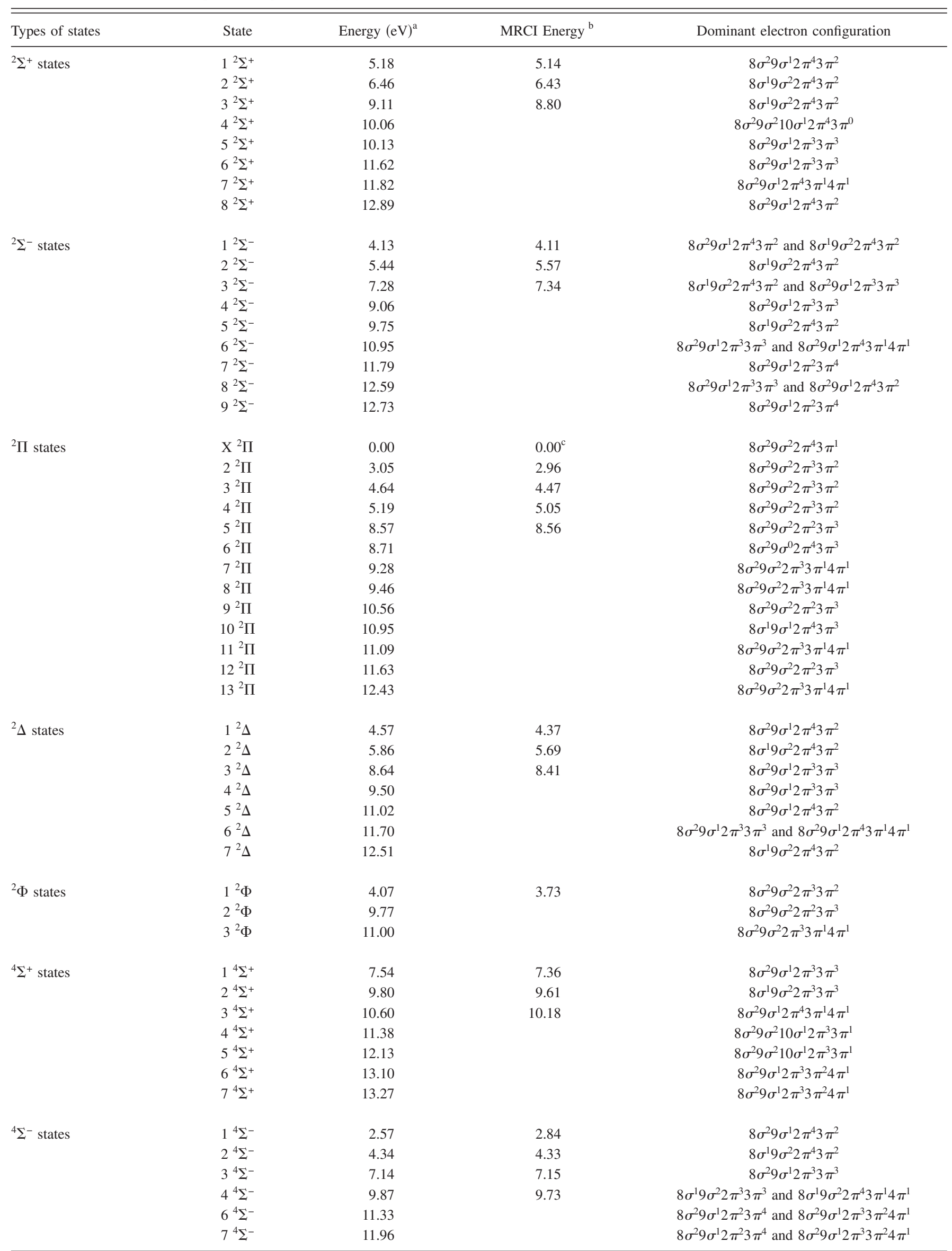


TABLE I. (Continued.)

\begin{tabular}{|c|c|c|c|c|}
\hline Types of states & State & Energy $(\mathrm{eV})^{\mathrm{a}}$ & MRCI Energy ${ }^{b}$ & Dominant electron configuration \\
\hline & $8^{4} \Sigma^{-}$ & 12.53 & & $8 \sigma^{2} 9 \sigma^{1} 2 \pi^{2} 3 \pi^{4}$ and $8 \sigma^{2} 9 \sigma^{1} 2 \pi^{3} 3 \pi^{2} 4 \pi^{1}$ \\
\hline & $9^{4} \Sigma^{-}$ & 12.75 & & $8 \sigma^{2} 9 \sigma^{2} 10 \sigma^{1} 2 \pi^{3} 3 \pi^{1}$ \\
\hline & $10^{4} \Sigma^{-}$ & 13.16 & & $8 \sigma^{1} 9 \sigma^{2} 2 \pi^{4} 3 \pi^{1} 4 \pi^{1}$ \\
\hline \multirow[t]{14}{*}{${ }^{4} \Pi$ states } & $1{ }^{4} \Pi$ & 2.54 & 2.41 & $8 \sigma^{2} 9 \sigma^{2} 2 \pi^{3} 3 \pi^{2}$ \\
\hline & $2{ }^{4} \Pi$ & 7.61 & 7.50 & $8 \sigma^{2} 9 \sigma^{2} 2 \pi^{3} 3 \pi^{1} 4 \pi^{1}$ and $8 \sigma^{2} 9 \sigma^{2} 2 \pi^{2} 3 \pi^{3}$ \\
\hline & $3{ }^{4} \Pi$ & 8.08 & 7.85 & $8 \sigma^{2} 9 \sigma^{2} 2 \pi^{3} 3 \pi^{1} 4 \pi^{1}$ \\
\hline & $4{ }^{4} \Pi$ & 8.82 & 8.68 & $8 \sigma^{1} 9 \sigma^{1} 2 \pi^{4} 3 \pi^{3}$ \\
\hline & $5{ }^{4} \Pi$ & 10.13 & 9.62 & $8 \sigma^{2} 9 \sigma^{2} 2 \pi^{3} 3 \pi^{1} 4 \pi^{1}$ \\
\hline & $6{ }^{4} \Pi$ & 11.59 & & $8 \sigma^{2} 9 \sigma^{2} 2 \pi^{3} 3 \pi^{1} 4 \pi^{1}$ and $8 \sigma^{2} 9 \sigma^{2} 2 \pi^{2} 3 \pi^{3}$ \\
\hline & $7{ }^{4} \Pi$ & 11.96 & & $8 \sigma^{2} 9 \sigma^{2} 10 \sigma^{1} 2 \pi^{4} 3 \pi^{1}$ and $8 \sigma^{2} 9 \sigma^{2} 2 \pi^{2} 3 \pi^{2} 4 \pi^{1}$ \\
\hline & $8{ }^{4} \Pi$ & 12.16 & & $8 \sigma^{2} 9 \sigma^{2} 10 \sigma^{1} 2 \pi^{4} 3 \pi^{1}$ and $8 \sigma^{2} 9 \sigma^{2} 2 \pi^{2} 3 \pi^{2} 4 \pi^{1}$ \\
\hline & $9{ }^{4} \Pi$ & 13.60 & & $8 \sigma^{1} 9 \sigma^{1} 2 \pi^{3} 3 \pi^{4}$ and $8 \sigma^{2} 9 \sigma^{0} 2 \pi^{4} 3 \pi^{2} 4 \pi^{1}$ \\
\hline & $10{ }^{4} \Pi$ & 13.69 & & $8 \sigma^{1} 9 \sigma^{1} 2 \pi^{3} 3 \pi^{4}$ \\
\hline & $11^{4} \Pi$ & 13.83 & & $8 \sigma^{2} 9 \sigma^{2} 2 \pi^{2} 3 \pi^{2} 4 \pi^{1}$ \\
\hline & $12{ }^{4} \Pi$ & 13.96 & & $8 \sigma^{2} 9 \sigma^{2} 2 \pi^{2} 3 \pi^{2} 4 \pi^{1}$ \\
\hline & $13^{4} \Pi$ & 14.11 & & $8 \sigma^{1} 9 \sigma^{2} 10 \sigma^{1} 2 \pi^{4} 3 \pi^{1}$ and $8 \sigma^{2} 9 \sigma^{1} 10 \sigma^{1} 2 \pi^{4} 3 \pi^{1}$ \\
\hline & $14{ }^{4} \Pi$ & 14.76 & & $8 \sigma^{1} 9 \sigma^{1} 2 \pi^{4} 3 \pi^{2} 4 \pi^{1}$ \\
\hline \multirow[t]{7}{*}{${ }^{4} \Delta$ states } & $1{ }^{4} \Delta$ & 7.28 & 7.32 & $8 \sigma^{2} 9 \sigma^{1} 2 \pi^{3} 3 \pi^{3}$ \\
\hline & $2{ }^{4} \Delta$ & 9.62 & 9.46 & $8 \sigma^{1} 9 \sigma^{2} 2 \pi^{3} 3 \pi^{3}$ \\
\hline & $3{ }^{4} \Delta$ & 10.58 & & $8 \sigma^{2} 9 \sigma^{1} 2 \pi^{4} 3 \pi^{2} 4 \pi^{1}$ and $8 \sigma^{1} 9 \sigma^{2} 2 \pi^{4} 3 \pi^{1} 4 \pi^{1}$ \\
\hline & $4{ }^{4} \Delta$ & 11.69 & & $8 \sigma^{1} 9 \sigma^{2} 2 \pi^{4} 3 \pi^{1} 4 \pi^{1}$ and $8 \sigma^{2} 9 \sigma^{1} 2 \pi^{3} 3 \pi^{2} 4 \pi^{1}$ \\
\hline & $5^{4} \Delta$ & 12.19 & & $8 \sigma^{2} 9 \sigma^{1} 2 \pi^{3} 3 \pi^{2} 4 \pi^{1}$ \\
\hline & $6{ }^{4} \Delta$ & 12.58 & & $8 \sigma^{2} 9 \sigma^{2} 10 \sigma^{1} 2 \pi^{3} 3 \pi^{1}$ \\
\hline & $7{ }^{4} \Delta$ & 13.64 & & $8 \sigma^{2} 9 \sigma^{1} 2 \pi^{3} 3 \pi^{2} 4 \pi^{1}$ \\
\hline \multirow[t]{2}{*}{${ }^{4} \Phi$ states } & $1{ }^{4} \Phi$ & 9.46 & 8.88 & $8 \sigma^{2} 9 \sigma^{2} 2 \pi^{3} 3 \pi^{1} 4 \pi^{1}$ \\
\hline & $2{ }^{4} \Phi$ & 12.98 & & $8 \sigma^{2} 9 \sigma^{2} 2 \pi^{2} 3 \pi^{2} 4 \pi^{1}$ \\
\hline
\end{tabular}

${ }^{\mathrm{a}}$ Total CASSCF energy at equilibrium geometry of OCS X ${ }^{1} \Sigma^{+}=-508.42672096 \mathrm{Eh}$.

${ }^{\mathrm{b}}$ MRCI results. See text for more details. Total MRCI energy at equilibrium geometry of OCS X ${ }^{1} \Sigma^{+}=-508.73498993 \mathrm{Eh}$.

${ }^{c}$ Used as reference.

$\left(\sim \mathrm{R}_{\mathrm{e}, \mathrm{CO}} \mathrm{OCS}^{3+} \mathrm{X}\right)$. Figure 7 displays the one-dimensional evolution of the doublets and quartets along the bending coordinate.

Close examination of these figures shows the following. (1) All states except the ground state are unbound in the C-S bond stretching direction. (2) A high density of electronic states is found along both the CO and CS coordinates. This high density allows the mixing of their wave functions by spin-orbit couplings (between the doublets and the quartets) at their respective crossings and vibronic interactions (between the electronic states having the same spin multiplicity) at their either crossings (between electronic states of different space symmetries) or avoided crossings (between electronic states of similar space symmetries). (3) The $\mathrm{OCS}^{3+}$ electronic states are mostly linear, except some quartets that may be stable at bent structures. (4) The shape of the potentials along the $\mathrm{CO}$ distance changes in response to changes in the CS distance. Especially, the ordering of some electronic states is modified by lengthening the CS distance from its equilibrium value in OCS X to its value in $\mathrm{OCS}^{3+} \mathrm{X}$. Hence, a very complex dynamics is expected for the unimolecular dissociation of the $\mathrm{OCS}^{3+}$ trications formed upon triple ionization of the neutral molecule. These fragmentation processes should evolve along the potentials of all electronic states depicted in these figures and involving their mutual couplings at both linear and bent structures.
To help interpret the experimental spectra, the energies of all states up to about $15 \mathrm{eV}$ above the ground state have been calculated at lower levels of theory, CASSCF and in some cases MRCI, at the equilibrium geometry of the neutral OCS molecule. There are about 80 states found in this energy range, including doublets and quartets with orbital symmetries $\Sigma^{+}, \Sigma^{-}, \Pi, \Delta$, and $\Phi$. As only four clear bands are seen in the spectra in the range of up to $15 \mathrm{eV}$ above the ground state, it is evident that all these 80 states are not equally likely to be populated and that a powerful selective process is at work in the double Auger effect. Because the double Auger spectra from inner shell holes on different atoms both in this (Fig. 2) and related cases ${ }^{20}$ differ considerably, electron location must be one significant factor.

As a working hypothesis, we propose that in the Auger effect, electrons are ejected principally from molecular orbitals (MOs) with their major density on the atom bearing the original charge. Simple MO calculations give the orbital characters, and the Mulliken atomic orbital populations have also been calculated before. ${ }^{19}$ The outermost orbital, $3 \pi$, is located almost exclusively on the $\mathrm{S}$ atom, the inner $2 \pi$ orbital is almost entirely on $\mathrm{O}$ and $\mathrm{C}$, the $9 \sigma$ orbital is again on $\mathrm{S}, 8 \sigma$ is on the $\mathrm{O}$ atom, and $7 \sigma$ is on $\mathrm{C}$ and $\mathrm{S}$. To use this information in practice, we select from the 80 states calculated to lie below $15 \mathrm{eV}$ excitation energy, as follows. 

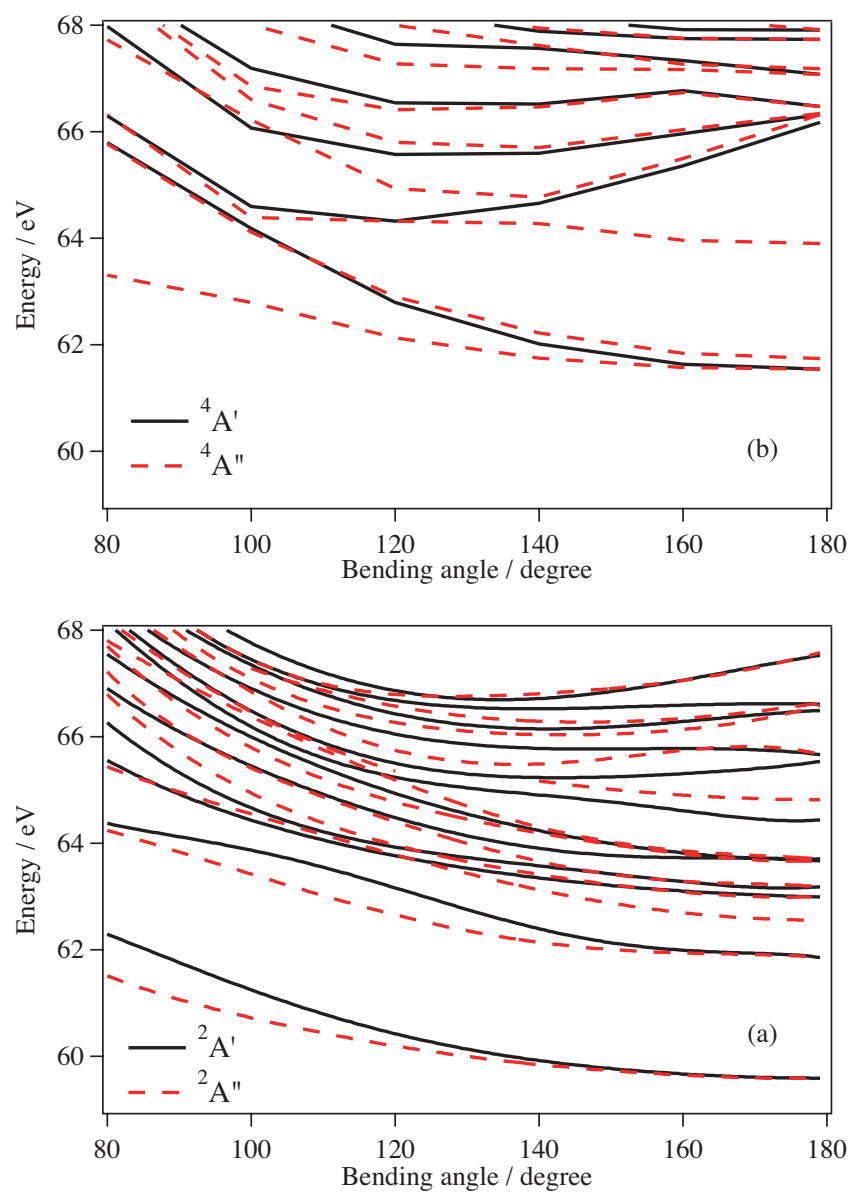

FIG. 7. CASSCF potential energy curves of the doublet and quartet electronic states of $\mathrm{OCS}^{3+}$ as a function of the bending angle ( $\theta$ in degrees). The $\mathrm{R}_{\mathrm{CO}}$ and the $\mathrm{R}_{\mathrm{CS}}$ distances are set to 2.175 and 3.58 bohrs, respectively. These curves are given in energy with respect to the OCS X ${ }^{1} \Sigma^{+}$minimum energy.

First, the complete list of 78 states is reduced to about half this number (43) by the selection of states with leading configurations derived only by electron ejection from the normally occupied orbitals. The exclusion of states requiring electron promotion for their formation seems reasonable, as transitions involving four electrons must be less probable than those involving only three. Second, the states are partitioned into those involving ejection of electrons from mainly S-based orbitals and from C-based ones. For sulfur this is straightforward, as the $3 \pi, 9 \sigma$, and $7 \sigma$ orbitals are dominantly S-based and 11 states, including the ground state, are formed by electron ejection from them. For carbon it is not so simple as there is only one calculated state in this energy range with a leading configuration not involving ejection of a $3 \pi$ electron. As a compromise the 17 states involving the $2 \pi$, $9 \sigma$, and $7 \sigma$ orbitals with not more than one electron removed from $3 \pi$ are selected. Finally, the question of doublet and quartet states arises. Because in single Auger from initially closed-shell species, doubly charged singlet states are formed more readily than triplet states, ${ }^{25,26}$ it seemed possible that a similar propensity may exist in double Auger. On account of this we have taken either pure doublets or both doublets and quartets on an equal footing in preparing the comparisons in

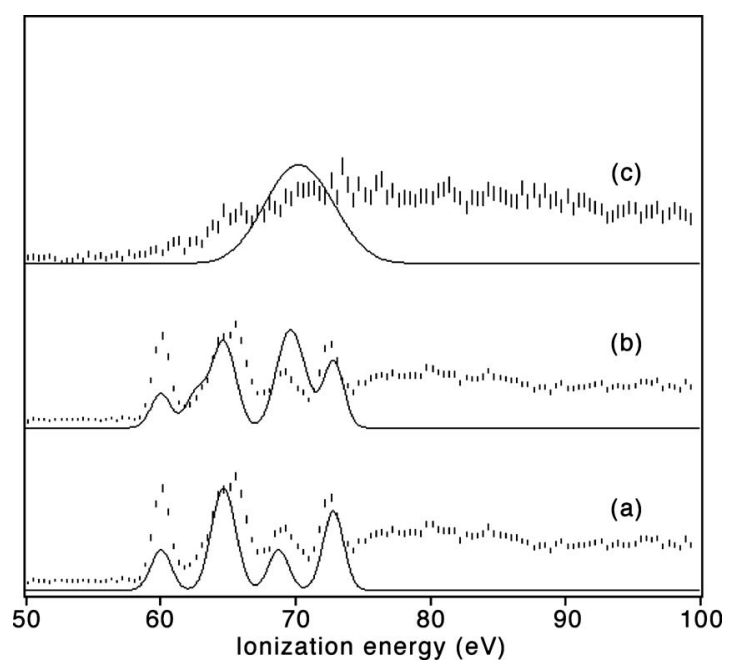

FIG. 8. Triple ionization spectra of OCS (error bars) compared with theoretical simulations as solid lines. (a) Spectrum from the $S 2 p$ hole with minimum $10 \mathrm{eV}$ Auger electron energy compared with simulated spectrum of selected doublet states only; (b) the same experimental spectrum compared with simulated spectrum of doublets and quartets; (c) spectrum from the $\mathrm{C} 1 \mathrm{~s}$ hole compared with a simulated spectrum of selected doublet states. See text for more details.

Fig. 8. Simulations were prepared by folding the selected state energies with Gaussian broadening functions of the expected instrumental width.

\section{DISCUSSION AND CONCLUSIONS}

The comparisons in Fig. 8 are striking. For the $\mathrm{S} 2 \mathrm{p}-$ related spectrum there is excellent agreement between the experimental spectrum and the theoretical one without quartet states, both in band positions and in relative intensities. When the quartets are included with the same weight as the doublets the match is very much worse. The conclusion that doublets are preferred over quartets in the double Auger effect, at least in this molecule, is inescapable. In the case of $\mathrm{C}$ $1 \mathrm{~s}$-induced triple ionization the theoretical simulation and the experimental spectrum match less well but not outrageously badly in view of the cut-off in calculated energies at $15 \mathrm{eV}$. Evidently much of the triple ionization via $\mathrm{C} 1 \mathrm{~s}$ populates higher energy states.

From the cumulative results and taking the spectrum fitting at face value, we can identify the states excited in the four main bands of the $\mathrm{S} 2 \mathrm{p}$-based spectrum of $\mathrm{OCS}^{3+}$. The band at $60 \mathrm{eV}$ represents the pure ground state ${ }^{2} \Pi$ state from removal of three electrons from the outermost $(3 \pi)$ orbital. The second band around $65 \mathrm{eV}$ comes from three ${ }^{2} \Sigma$ states where two electrons are missing from $3 \pi$ and one is missing from $9 \sigma$. The third band, around $69 \mathrm{eV}$, is mainly a ${ }^{2} \Pi$ state where two electrons are gone from $9 \sigma$ and only one from $3 \pi$. The fourth band, near $72 \mathrm{eV}$, originates from ${ }^{2} \Sigma$ states from removal of one $9 \sigma$ and two $3 \pi$ electrons. This analysis is confirmed by the selective effects of selecting molecularfield-split components of the originating $S 2 p$ hole state. From analysis of the single Auger spectrum ${ }^{27}$ of $\mathrm{HCl}$ and similar but even stronger effects in that of $\mathrm{HBr}^{28}$ it is known that in the single Auger effect, $\sigma$-electrons are ejected much more strongly from $\Sigma$-like $(\Omega=1 / 2)$ initial holes than from 
I-like $(\Omega=3 / 2)$ holes. This is exactly the pattern of behavior seen in Fig. 4 in the present double Auger effect where the ground state (pure $\pi^{-3}$ ) is relatively much stronger than the $\Omega=3 / 2$ (П) initial hole, while the higher $\sigma$-derived states are stronger than $\Omega=1 / 2(\Sigma)$. This is certainly the first time that the spectrum of any triply ionized molecule has been observed and analyzed in such detail.

In addition to the spectroscopy, we can draw tentative conclusions about the general propensities of triple ionization by the double Auger effect. First, localization effects are a major and possibly the dominant factor in determining the choice of triply ionized states to be populated. A similar conclusion relative to single Auger was drawn from the normal Auger spectrum of this same molecule long ago. ${ }^{19}$ Second, as mentioned above, doublets are probably preferred over quartets in the double Auger effect from initially closed shells. Third, the notably improved spectral sharpness obtained in the double Auger spectra by eliminating extreme asymmetric energy sharing between the Auger electrons is mainly an effect of the instrumental resolution, though additional effects including cascade Auger pathways are probably also in play, particularly for band intensities. Finally, for linear molecules $\Sigma$-like $(\Omega=1 / 2)$ initial holes eject $\sigma$ electrons preferentially in the double Auger effect, just as they do in the single Auger effect.

\section{ACKNOWLEDGMENTS}

This work has been financially supported by the Swedish Research Council (VR), the Göran Gustafsson Foundation (UU/KTH), the Knut and Alice Wallenberg Foundation, and the Wenner Gren Foundation, Sweden. J.H.D.E. thanks the Leverhulme Trust for financial support. We are also grateful for the technical support of the workshop staff at the AlbaNova Research Centre in Stockholm as well as at the Ångström Laboratory in Uppsala while adapting the experimental setup to synchrotron radiation. Furthermore, we would like to warmly acknowledge the support by the staff and colleagues at BESSY, Berlin. This work was also supported by the European Community-Research Infrastructure Action under the FP6 "Structuring the European Research Area" Programme (through the Integrated Infrastructure Initiative "Integrating Activity on Synchroton and Free Electron Laser Science"-Contract No. R II 3-CT-2004-506008).

${ }^{1}$ A. S. Newton, J. Chem. Phys. 40, 607 (1964).

${ }^{2}$ P. Lablanquie, L. Andric, J. Palaudoux, U. Becker, M. Braune, J. Viefhaus, J. H. D. Eland, and F. Penent, J. Electron Spectrosc. Relat. Phenom. 156-158, 51 (2007).

${ }^{3}$ Y. Hikosaka, P. Lablanquie, F. Penent, T. Kaneyasu, E. Shigemasa, J. H. D. Eland, T. Aoto, and K. Ito, Phys. Rev. A 76, 032708 (2007).

${ }^{4}$ J. H. D. Eland, P. Linusson, L. Hedin, E. Andersson, J.-E. Rubensson, and R. Feifel, Phys. Rev. A 78, 063423 (2008).

${ }^{5}$ Y. Hikosaka, P. Lablanquie, F. Penent, T. Kaneyasu, E. Shigemasa, R. Feifel, J. H. D. Eland, and K. Ito, Phys. Rev. Lett. 102, 013002 (2009).

${ }^{6}$ S. M. Thurgate, J. Electron Spectrosc. Relat. Phenom. 81, 1 (1996).

${ }^{7}$ F. Penent, P. Lablanquie, R. I. Hall, J. Palaudoux, K. Ito, Y. Hikosaka, T. Aoto, and J. H. D. Eland, J. Electron Spectrosc. Relat. Phenom. 144147, 7 (2005).

${ }^{8}$ M. Hochlaf, G. Chambaud, and P. Rosmus, J. Chem. Phys. 108, 4047 (1998).

${ }^{9}$ V. Brites, J. H. D. Eland, and M. Hochlaf, Chem. Phys. 346, 23 (2008).

${ }^{10}$ J. H. D. Eland, O. Vieuxmaire, T. Kinugawa, P. Lablanquie, R. I. Hall, and F. Penent, Phys. Rev. Lett. 90, 053003 (2003).

${ }^{11}$ J. H. D. Eland and R. Feifel, Chem. Phys. 327, 85 (2006).

${ }^{12}$ See: http://www.molpro.net.

${ }^{13}$ T. H. Dunning, J. Chem. Phys. 90, 1007 (1989).

${ }^{14}$ D. E. Woon and T. H. Dunning, J. Chem. Phys. 98, 1358 (1993).

${ }^{15}$ P. J. Knowles and H.-J. Werner, Chem. Phys. Lett. 115, 259 (1985).

${ }^{16}$ H.-J. Werner and P. J. Knowles, J. Chem. Phys. 89, 5803 (1988).

${ }^{17}$ P. J. Knowles and H.-J. Werner, Chem. Phys. Lett. 145, 514 (1988).

${ }^{18}$ P. J. Knowles, C. Hampel, and H.-J. Werner, J. Chem. Phys. 99, 5219 (1993); 112, 3106(E) (2000).

${ }^{19}$ T. X. Carroll, D. Ji, and T. D. Thomas, J. Electron Spectrosc. Relat. Phenom. 51, 471 (1990).

${ }^{20}$ P. Linusson, J. H. D. Eland, E. Andersson, L. Hedin, J.-E. Rubensson, and R. Feifel (unpublished).

${ }^{21}$ T. N. Chang and R. T. Poe, Phys. Rev. A 12, 1432 (1975).

${ }^{22}$ J. H. D. Eland, Adv. Chem. Phys. 141, 103 (2009).

${ }^{23}$ R. Feifel, J. H. D. Eland, and D. Edvardsson, J. Chem. Phys. 122, 144308 (2005).

${ }^{24}$ D. M. P. Holland and M. A. MacDonald, Chem. Phys. 144, 279 (1990).

${ }^{25}$ H. Ågren, J. Chem. Phys. 75, 1267 (1981).

${ }^{26}$ F. Tarantelli, A. Sgamellotti, and L. S. Cederbaum, in Applied ManyBody Methods in Spectroscopy and Electronic Structure, edited by D. Mukherjee (Plenum, New York, 1992).

${ }^{27}$ R. F. Fink, Phys. Rev. A 58, 1988 (1998).

${ }^{28}$ P. Lablanquie and F. Penent, personal communication (July 2009). 